\title{
Prosthetic Visualizations for a Smart Heritage
}

\author{
Stefano Brusaporci \\ Pamela Maiezza \\ Alessandra Tata \\ Fabio Graziosi \\ Fabio Franchi
}

\section{Abstract}

The development of ICT has favoured the spread of real-time, pervasive and ubiquitous applications. In particular, VR and AR visualizations allow a close interrelation between people, data, environments and objects. Consequently, it is possible to enrich cultural heritage with information by visually superimposing multimedia content, in absolute respect of their physical consistency. In this way, it is possible to create a 'smart heritage' dimension that combines the potential of the 'Phygital' with the protection and enhancement of assets often characterized by important elements of fragility. An important role is played by $\mathrm{Al}$ applications, which automatically direct the processes of 'Interpretation' and 'Presentation' of the heritage. Based on the experience of the 3D reconstruction of the no longer existing Baroque configuration of the Basilica of Collemaggio in L'Aquila, aim of the paper is a theoretical-methodological reflection on the concept of VR / AR / MR for cultural heritage.

\section{Keywords}

augmented reality, architectural heritage, 3D modelling, smart heritage, digital heritage.

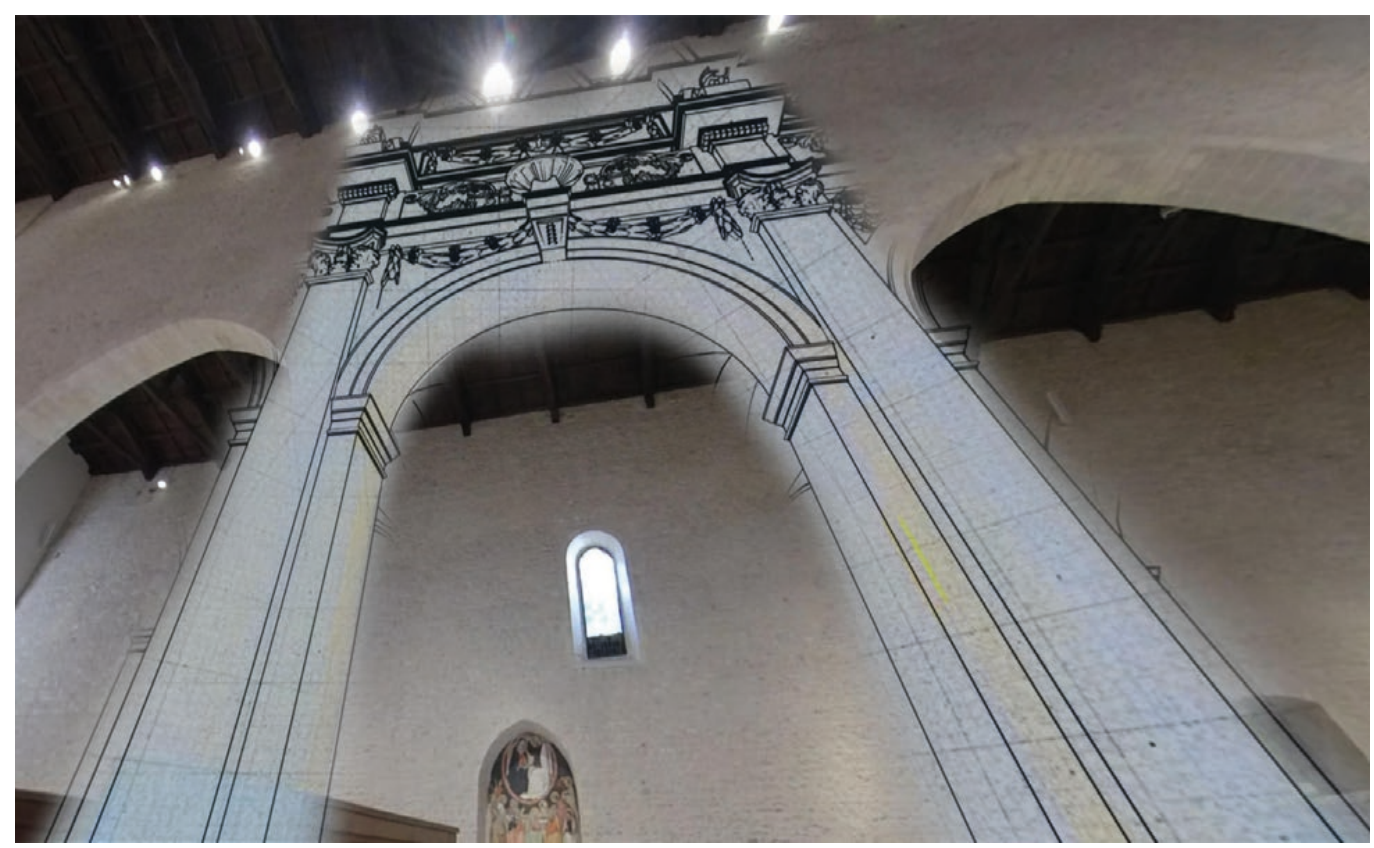




\section{Introduction}

The development of ICT has favoured the spread of real-time, pervasive and ubiquitous applications, which allow the interrelation between information, people and environments. In this way, thanks to smart devices, each user is constantly immersed in an on-life state [Floridi 20I5]. In this context, Artificial Intelligence (Al), combining data, algorithms and computational power through machine learning processes, plays a very important role. In particular, the White Paper on Artificial Intelligence: a European approach to excellence and trust [European Commission 2020] highlights the potential of Al for citizens, economic activities and the public good: "the impact of Al systems should be considered not only from an individual perspective, but also from the perspective of society as a whole. The use of Al systems can have a significant role in achieving the Sustainable Development Goals, and in supporting the democratic process and social rights [...] Promoting the adoption of Al by the public sector [...for an...] An ecosystem of excellence". In the field of cultural heritage, Al can significantly influence the processes of interpretation and presentation underlying the conservation and enhancement of cultural heritage [Tielden 1957; ICOMOS 2008], playing a leading role through user profiling with repercussions in the methods of storytelling and, above all, the mechanisms of interaction and participation. The interaction between virtual reality, augmented reality, mixed reality and artificial intelligence can lead to direct consequences in the context of the methods of defining and declining experiences in the context of digital heritage [Brusaporci 2017; Pierdicca et al. 2020].

The paper presents a theoretical-methodological reflection on the concept of smart heritage starting from VR/AR/MR visualizations for cultural heritage, which is based on an experience related to the $3 \mathrm{D}$ digital reconstruction of the no longer existing baroque configuration of the Basilica of Collemaggio in L'Aquila (figs. I, 2) [Brusaporci et al. 202 I] [I].

\section{Smart Visualizations}

The traditional static approach to 'smart cities', centered on infrastructural networks, has developed in the sense of a cultural dimension, where the concept of 'smart cities' has evolved into that of 'smart places', in particular thanks to participatory declinations. Operationally, 'smartness' requires the integration of 'objects' with sensors and their enrichment with information, so that they can interact with the environment. If the so-called 'Internet of Things' poses problems of a substantially technological nature if applied to contemporary products, when it is addressed to artefacts characterized by historical and aesthetic values, issues of a theoretical-methodological nature arise. These relate both to the image of the work and to the respect for its materiality, testimony to the events and cultures that have taken place over time. Therefore, it follows the need for precise reflections on the methods that can favour the information enrichment of cultural heritage. The wellknown principles of documentation, recognisability, reversibility and protection, in particular when interacting with the physicality of the assets - for example with sensors or targets - remain firm. One solution is to interact with the artifact only in the visual field, through temporary and mediated views that enrich it with information. This is the case

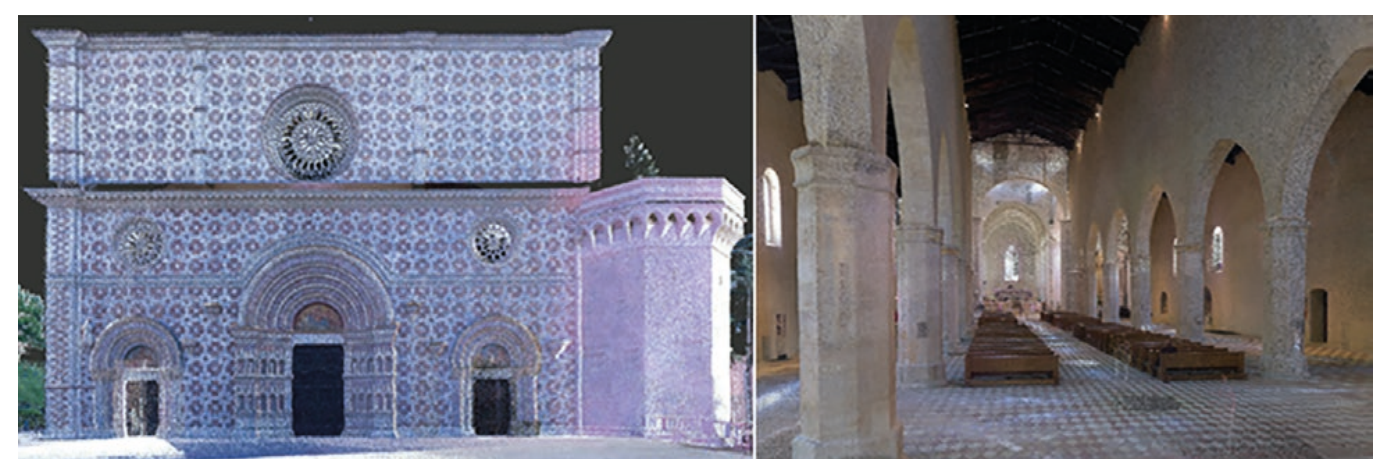


of video mapping [Rossi 2013], but above all it is what is offered by augmented reality applications [Ch'ng et al. 2017; Clini et al. 2017], able to automatically recognize what is framed by the camera of the device and to superimpose information of various kinds. This type of experimentation is conducted by the research group of the University of L'Aquila, starting from the INCIPICT project [http://incipict.univaq.it/] integrated with the 5G experimentation, through a proprietary app specially developed for the enhancement of cultural, architectural and urban heritage of L'Aquila [Brusaporci et al.20 I9].The application, which can be used in urban areas of the city and in specific interiors, is implemented to tell the history of monuments and places, also by superimposing in real-time 3D virtual reconstructions of past configurations that no longer exist (figs 3, 4). Specifically, the paper presents the experience dedicated to the Basilica of Collemaggio, the heart of the rite of 'Perdonanza', which was included into the UNESCO Intangible Cultural Heritage Lists in 2019. Furthermore, the $A R$ app opens to lines of research aimed at $\mathrm{Al}$, with for example image recognition, user profiling, prediction and management of contents and interactions [Pierdicca et al. 2020]. Conceptually, the close interaction between real heritage - experienced in its own historical environment - and digital visualization refers to the concept of 'Phygital' [Nolaf 2019] and strengthens its applications in the field of cultural heritage.

\section{The AR App by Univaq}

Mobile augmented reality (Mobile AR) is gaining increasing attention from both academia and industry. Hardware-based Mobile AR and App-based Mobile AR are the two dominant platforms. However, hardware-based Mobile AR implementation is known to be costly and lacks flexibility, while the App-based one requires additional downloading and installation in advance and is inconvenient for cross-platform deployment. However, with the improved communication and computation capabilities provided by $5 \mathrm{G}$ technologies, a combination of both technologies is growing up in order to support tourists and cultural applications. Furthermore, the emergence of $5 \mathrm{G}$ mobile communication networks has the potential to enhance the communication efficiency of Mobile AR dense computing in the MEC approach [2]. Several technological advances have started to enter the landscape of Mobile AR. First, the upcoming 5G networks [Coluccelli et al. 20I8] bring new opportunities for Mobile AR. They provide higher bandwidth $(0.1 \sim \mid \mathrm{Gb} / \mathrm{s})$ and lower network delay $(I \sim 10 \mathrm{~ms})$, which improves the data transmission on mobile networks. Second, the introduction of new characteristics, such as MEC, device-to-device (D2D) communication, and network slicing, provides an adaptive and scalable communication mechanism that further provides efficient infrastructures for the deployment and promotion of Mobile AR.

Within the INCIPICT project a MEC based demonstration testbed has been set-up. The system exploits the platform available at the MEC LAB of the University of L'Aquila in order to validate the capabilities of the MEC architecture to support applications dedicated to AR services. The MEC LAB provides a complete and customizable network environment

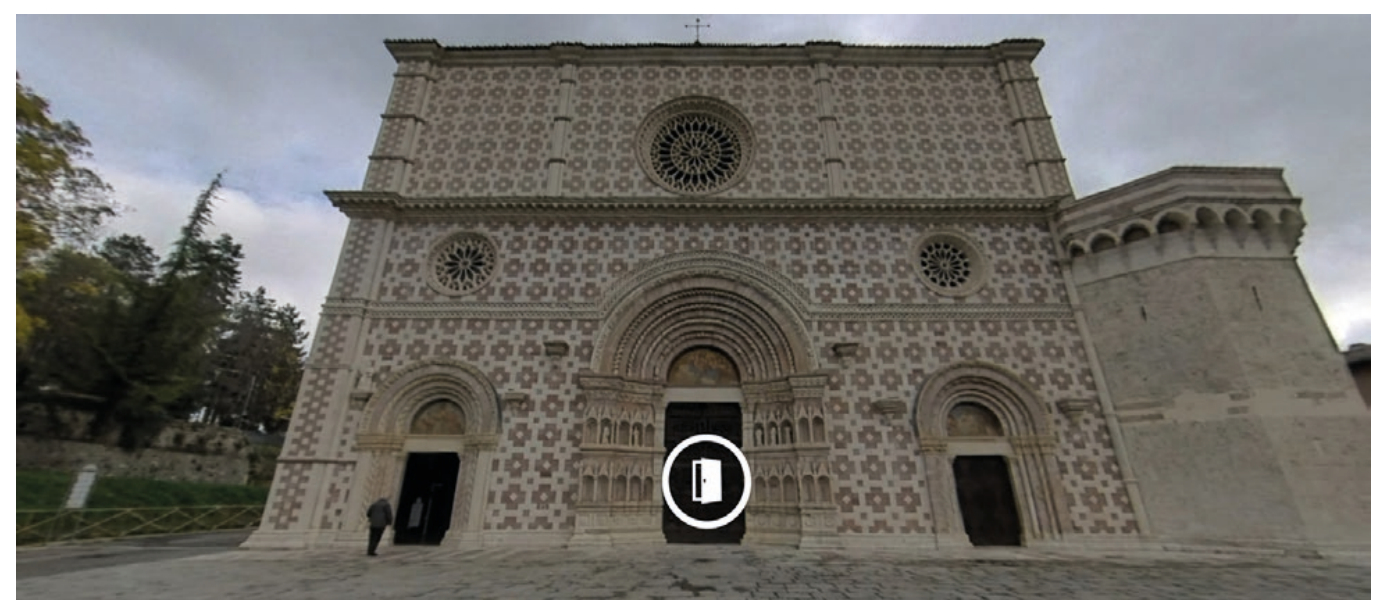


Fig. 3. Screenshot of the Collemaggio AR application: reconstruction of the baroque ceiling and superimposition with the current configuration.
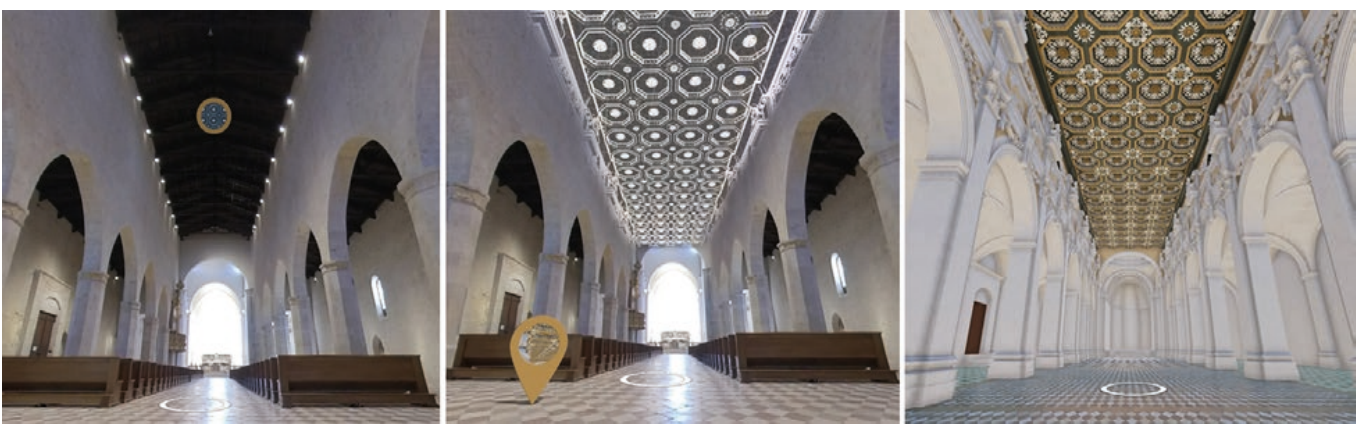

and consists of 3 nodes distributed in the city of Aquila. In the University hub, more than I5 physical servers are available and interconnected using optical and wireless technologies to provide heterogeneous connectivity between nodes up to l0Gbps per network segment. The laboratory hosts also a $5 \mathrm{G}$ radio access network and a core network to implement network slicing with guaranteed performance on a common physical infrastructure and it is used to perform edge computing experiments. The availability of distributed computing infrastructures in the city allows the experimentation of the orchestration of virtual services in metropolitan networks required by the AR services.

Moreover, a mobile app based on Apple's ARKit has been developed in order to evaluate service. In order to exploit the system some 3D models were created in different sizes. AR was made by the developed application available for $5 \mathrm{G}$ smartphones. All 3D models were stored into the MEC platform and accessed by the $5 \mathrm{G}$ network minimizing the latency and with a very high throughput in order to provide the best user experience. Once available within the app a user can browse the 3D model using the device camera.

\section{Conclusion}

The examples offered by Google and Amazon to all users highlight how mixed reality applications are and will be increasingly widespread. Therefore, it is believed that the analysis of the possibilities offered in the field of knowledge, conservation and enhancement of cultural heritage is of great importance. The experiment presented is only a first step within a line of research dedicated to the study of architectural heritage.

We want to conclude with a general reflection on the digital dimension of the visual in the narration of cultural heritage, starting from the well-known "Uncanny Valley" by Masahiro Mori, that is, his graphs dedicated to the sense of affinity of people towards robots, in relation their resemblance to humans and their ability to move. We could think of updating Mori's reflection by replacing robots with digital visualizations (VR, $A R, M R$ ), and the concept of 'movement' by the influence of machine learning, able to simulate our behaviour in an increasingly effective way and to prevent our requests and wishes more and more efficiently. In the current
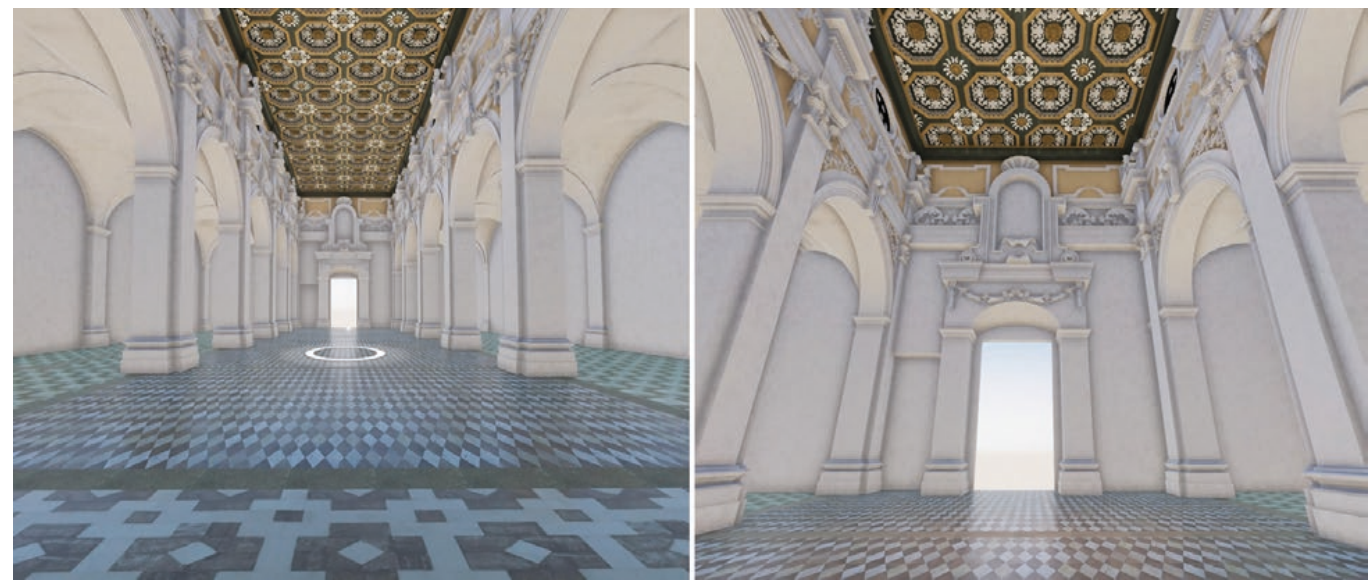
so-called "Age of Culture" [Schafer 2014], ethical questions arise where Human Computer Interaction can prefigure new reflections and experiments aimed at the interpretation and presentation of cultural heritage. In this context, Al plays a leading role, where users are increasingly accustomed to the use of synthetic and cunning images in the relationship between real and digital [Brusaporci 2019].

\section{Notes}

[I] The research has received funding from the Italian Government under Cipe resolution n. I 35 (Dec. 2I, 20I2), project INnovating City Planning through Information and Communication Technologies (INCIPICT).

[2] MEC, formerly mobile edge computing, refers to the enabling technologies that provide computing capabilities and service environment at the edge of the network [European Telecommunications Standards Institute (ETSI) White Paper].

\section{References}

Brusaporci Stefano (2017). Digital Innovations in Architectural Heritage Conservation: Emerging Research and Opportunities. Hershey (PA): IGI Global.

Brusaporci Stefano, Graziosi Fabio, Franchi Fabio, Maiezza Pamela (2019). Remediating the Historical City. Ubiquitous Augmented Reality for Cultural Heritage Enhancement. In Luigini Alessandro (ed.). Proceedings of the Ist International and Interdisciplinary Conference on Digital Environments for Education, Arts and Heritage EARTH 20 I 8. Cham: Springer, pp. 305-31 3.

Brusaporci Stefano (2019). The visual bride: representing tangible heritage between digitality and real contents. In Luigini Alessandro, Panciroli Chiara (eds.). img journal $01 / 2019$ Manifesto. vol. O I. Alghero: Publica, pp. 74-91.

Brusaporci Stefano, Graziosi Fabio, Franchi Fabio, Maiezza Pamela, Tata Alessandra (202I). Mixed Reality Experiences for the Historical Storytelling of Cultural Heritage. In Bolognesi Cecilia, Villa Daniele (eds.). From Building Information Modelling to Mixed Reality. Cham: Springer, pp. 33-46.

Ch'ng Eugene, Cai Yiyu, Thwaites Harold (Eds.) (2017). Special Issue on VR for Culture and Heritage: The Experience of Cultural Heritage with Virtual Reality: Guest Editors' Introduction. In PRESENCE:Virtual and Augmented Reality, 26(3), pp. iii-vi.

Clini Paolo, Quattrini Ramona, Frontoni Emanuele, Pierdicca Roberto, Nespeca Romina (2017). Real/not real: pseudoholography and augmented reality applications for cultural heritage. In Handbook of Research on Emerging Technologies for Digital Preservation and Information Modeling. Hershey (PA): IGI Global, pp. 20 I-227.

Coluccelli Gabriella, Loffredo Vincenzo, Monti Luca, Spada Maria Rita, Franchi Fabio, Graziosi Fabio (20 I8). 5G Italian MISETrial: Synergies Among Different Actors to Create a "5G Road". In 2018 IEEE 4th International Forum on Research and Technology for Society and Industry (RTSI). Marsiglia: Institute of Electrical and Electronics Engineers (IEEE) Publisher, pp. I-4.

Floridi Luciano (ed.) (20 I5). The Onlife Manifesto. Being Human in a Hyperconnected Era. Cham: Springer.

ICOMOS (2008). Charter for the Interpretation and Presentation of Cultural Heritage Sites. http://icip.icomos.org/downloads/ ICOMOS_Interpretation_Charter_ENG_04_10_08.pdf (I 5 January 2021).

Mori Masahiro ( 1970). Bukimi no tani - The uncanny valley. (K. F. MacDorman \& T. Minato, Trans.). Energy, 7(4), 33-35. https:// web.ics.purdue.edu/ drkelly/MoriTheUncannyValley1970.pdf (I 5 January 202 I).

Nolaf Eslam (2019). Phygital Heritage. Communicating Built Heritage Information through the Integration of Digital Technology into Physical Reality. PhD Thesis, KU Leuven.

Pierdicca Roberto, Paolanti Marina, Frontoni Emanuele, Baraldi Lorenzo (2020). Al4AR: An Al-Based Mobile Application for the Automatic Generation of AR Contents. In De Paolis Lucio Tommaso, Bourdot Patrick (eds.). Augmented Reality, Virtual Reality, and Computer Graphics. AVR 2020. Lecture Notes in Computer Science, vol. 12242. Cham: Springer.

Rossi Daniele (20I3). Smart architectural models. Spatial projection based augmented mock-up. In Addison Alonzo C., Guidi Gabriele, De Luca Livio, Pescarin Sofia (eds.). 2013 Digital Heritage International Congress, vol. 2. Marsiglia: Institute of Electrical and Electronics Engineers (IEEE) Publisher.

Schafer D. Paul (20I4). The Age of Culture. Oakville (ON): Rock's Mills Press.

Tielden Freeman (1957). Interpreting Our Heritage. Chapel Hill: University of North Carolina Press.

\section{Authors}

Stefano Brusaporci, Dept. of Civil, Construction-Architectural and Environmental Engineering, University of L'Aquila, stefano.brusaporci@univaq.it Pamela Maiezza, Dept of Civil, Construction-Architectural and Environmental Engineering, University of L'Aquila, pamela.maiezza@univaq.it Alessandra Tata, Dept. of Civil, Construction-Architectural and Environmental Engineering, University of L'Aquila, alessandra.tata@graduate.univaq Fabio Graziosi, Dept. of Information Engineering, Computer Science and Mathematics, University of L'Aquila, fabio.graziosi@univaq.it Fabio Franchi, Dept. of Information Engineering, Computer Science and Mathematics, University of L'Aquila, fabio.franchi@univaq.it 
